\title{
Acute spinal subdural hematoma complicating lumbar decompressive surgery
}

Authors Kok Chun Chang, Dino Samartzis, Keith DK Luk, Kenneth MC Cheung, Yat-Wa Wong

Institution Department of Orthopaedic \& Traumatology, Queen Mary Hospital, University of Hong Kong,

Pokfulam, Hong Kong SAR, China

\section{ABSTRACT}

Study design: A case report.

Objective: To report a rare case of acute spinal subdural hematoma ( $\mathrm{SSH}$ ) complicating lumbar spine surgery, its characteristic presenting symptoms, diagnostic imaging, possible cause, and pitfall in management.

Methods: A 59-year-old woman with lumbar spinal instability and stenosis underwent laminectomy and decompression at L3-L5 with instrumentation and fusion from L3-S1.

Results: Immediately following surgery, the patient presented with incapacitating pain of both lower extremities from the mid-thigh downward, which was not relieved by narcotic analgesia and was disproportional to surgical trauma. Left ankle and great toes weakness was detected at postoperative day 2 and deteriorated on day 6. Magnetic resonance imaging was performed urgently and revealed a characteristic SSH with thecal sac compression at the level of L2, proximal to the laminectomy. Emergency decompression and evacuation of the hematoma was performed. The patient had partial recovery 6 weeks postoperatively.

Conclusion: Acute SSH is a rare complication of lumbar spine surgery. This diagnosis must be considered when severe leg pain, unresolved with analgesia and disproportional to surgical trauma, with neurological deterioration occurring after lumbar spine surgery. Magnetic resonance imaging is the imaging modality of choice to assist in the differential diagnosis of an SSH. Early surgical decompression is necessary for optimal neurological recovery.

The authors have no competing or financial interests to disclose in relation to this work. 


\section{INTRODUCTION}

Spinal subdural hematoma (SSH) is a rare cause of spinal neutral element compression. Such a lesion typically manifests in patients with vascular malformations, tumors, bleeding disorders, anticoagulant therapy, trauma, or infection [1-5]. Also, the development of SSH has also been associated with diagnostic lumbar puncture and spinal anesthesia $[6,7]$.

Spinal subdural hematoma is a rare complication of spine surgery, with only few cases of subacute presentation reported in the literature associated with surgical trauma [ 1 , 2]. However, little is known regarding the manifestation of an acute SSH following lumbar spine surgery. As such, the authors report their experience in the diagnosis and management of an acute SSH, which presented immediately after lumbar decompression and fusion surgery.

\section{CASE REPORT}

A 59-year-old woman without significant past medical problems was admitted to the orthopaedic clinic complaining of mechanical low back pain and left lower extremity sciatica, which radiated to the left buttocks and lateral calf regions. She had a positive left straight-leg raising test. There was sensory impairment of the left L5 dermatomes but without motor weakness of both lower extremities. Her walking distance was limited to less than 5 minutes

Fig 1a-b Preoperative (a) lateral and (b) posteroanterior plain x-rays illustrating severe degenerative changes of the lower lumbar spine region with grade 1 spondylolisthesis of L3-L4 and L5-S1.
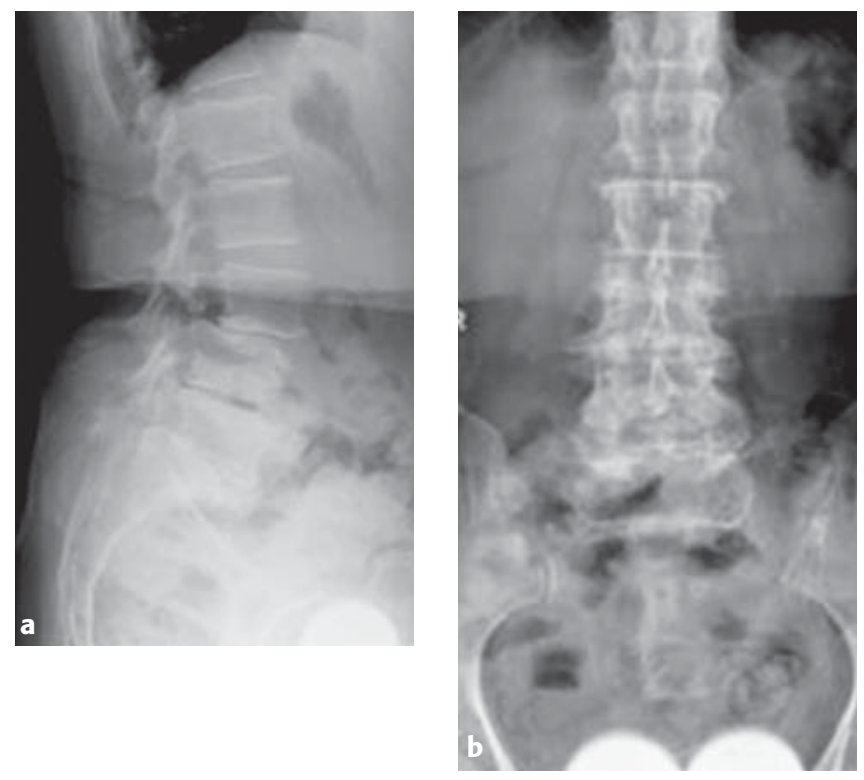

on level ground because of the increasing left L5 pain and numbness.

Plain $x$-rays of the lumbo-sacral spine showed degenerative changes of lower lumbar region with unstable grade 1 spondylolisthesis of L3-L4 and L5-S1 (Fig 1). Magnetic resonance imaging (MRI) of the lumbosacral spine also noted degenerative lumbar spinal stenosis of L3-L4, L4-L5 and L5-S1, with no suspicious lesions of vascular malformation (Fig 2).

The patient reported no improvement with prolonged course of conservative treatment. Surgery was then performed, which consisted of a wide laminectomy with bilateral nerve root decompression of L3-L5, pedicle screw instrumentation from L3-S1, and inter-transverse fusion by autogenous bone graft harvested from the iliac crest (Fig 3). Intraoperatively, the dura was found to be adherent at L3-L4 and L5-S1 levels, and it was bluntly dissected off the lamina without violation of the dura. The fusion construct was made without intention of reduction of the spondylolisthesis. The operation lasted 4 hours and 25 minutes. The systolic blood pressure was kept low throughout surgery, and there was no excessive bleeding suggestive of coagulopathy.

On postoperative day 1 , the patient complained of severe pain of both lower extremities from mid-thigh downward. The pain was not controlled even by frequent high-dose postoperative parenteral narcotic analgesic. In contrast, there was minimal surgical wound pain. She was not able to cooperate with neurological assessment because of narcotic sedation and severe pain. Physical examina-

Fig 2 Sagittal T2-weighted MRI demonstrating spinal stenosis of L3-L4, L4-L5, and L5-S1 levels with disc degeneration and endplates changes.

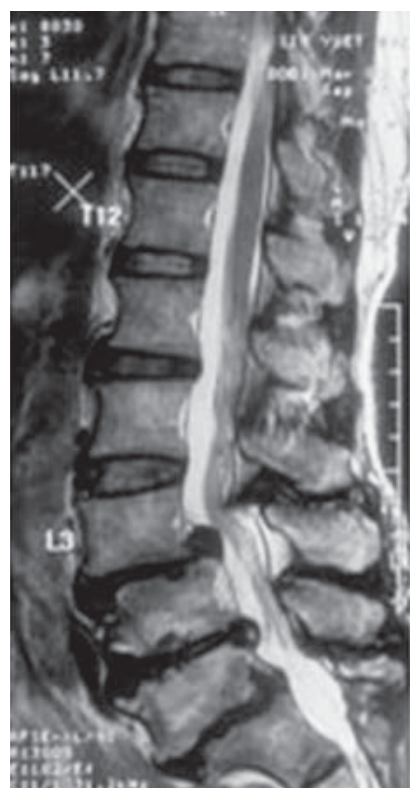


tion on the following day revealed grade 4 weakness of left ankle dorsiflexion (L4) and bilateral grade 3 great toe extensions (L5). Her rectal examination revealed an intact perianal sensation and anal tone. The possibility of a caudal equina compression by hematoma was believed unlikely due to the presence of a functioning deep drain. Residual spinal stenosis was also believed to be unlikely since a wide laminectomy was performed with thorough nerve root decompression. Pedicle screws were inserted under direct vision after the wide laminectomy, and the postoperative $\mathrm{x}$-rays did not suggest screw malposition or poor alignment. It was concluded that intraoperative dural dissection and manipulation of the neural tissue had caused a neurapraxic lesion. On postoperative day 3, leg pain receded to the mid-leg region and distally but the weakness persisted.

On postoperative day 6 , neurological deterioration was detected with grade 4 weakness of great toes flexion, grade 3 great toes extension, and grade 2 left-sided ankle dorsiflexion. In view of worsening neurology, an MRI scan was performed (Fig 4). T2-weighted sagittal MRI showed a bright signal subdural hematoma proximal to the laminectomy site at the level of L2, causing cauda equina compression (Fig 4a). Blood-soaked gel foams were noted at L3, L4, and at the L5 laminectomy site with no neural compression. T2-weighted axial MRI showed bright signal intensity, representative of a lentiform subdural hematoma of the left posterolateral region of the spinal canal (Fig $\mathbf{4 b}$ ). The hematoma was confined to the dura and did not extend into the bony recess.

Emergency exploration was performed on postoperative day 6. A partial L2 laminectomy was performed. The dura appeared unremarkable but a durotomy revealed a collection of xanthomatic fluid, which was compatible with a lysed hematoma. The dura was repaired by $6 / 0$ nonabsorb-

Fig 3a-b Immediate postoperative (a) lateral and (b) posteroanterior plain $x$-rays illustrating laminectomy of L3-L5 and intact instrumentation from L3-S1.
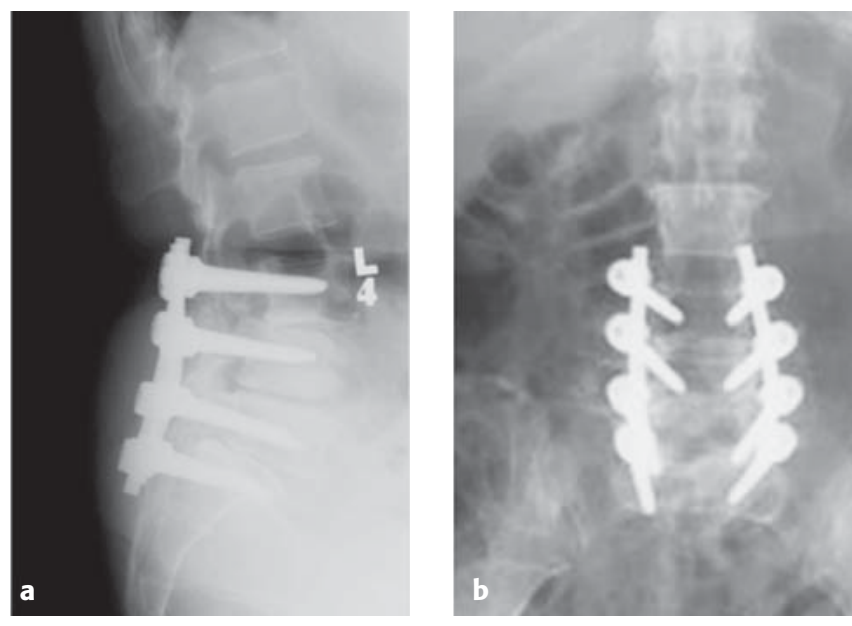

able suture. Postoperative MRI confirmed decompression of the cauda equina and disappearance of SSH (Fig 5). Six weeks following surgery, the patient reported persistent bilateral paraesthesia below the level of the mid-legs. The sensation was reduced from L2 downward with pin and needle paraesthesia from the mid-leg and below. She could walk independently with a cane.

Fig 4a-b T2-weighted magnetic resonance imaging. a Sagittal image demonstrating hyperintense signal intensity at L2 level suggestive of a subdural hematoma causing cauda equina compression (upper arrow). Blood-soaked gel foams are noted at the laminectomy site of L3-L5 (lower arrow). b Axial imaging of L2 showing a hyperintense signal intensity, correlating with a subdural hematoma at the left posterolateral location of the spinal canal (arrow). It was confined within the dura with no extension into the lateral recess.
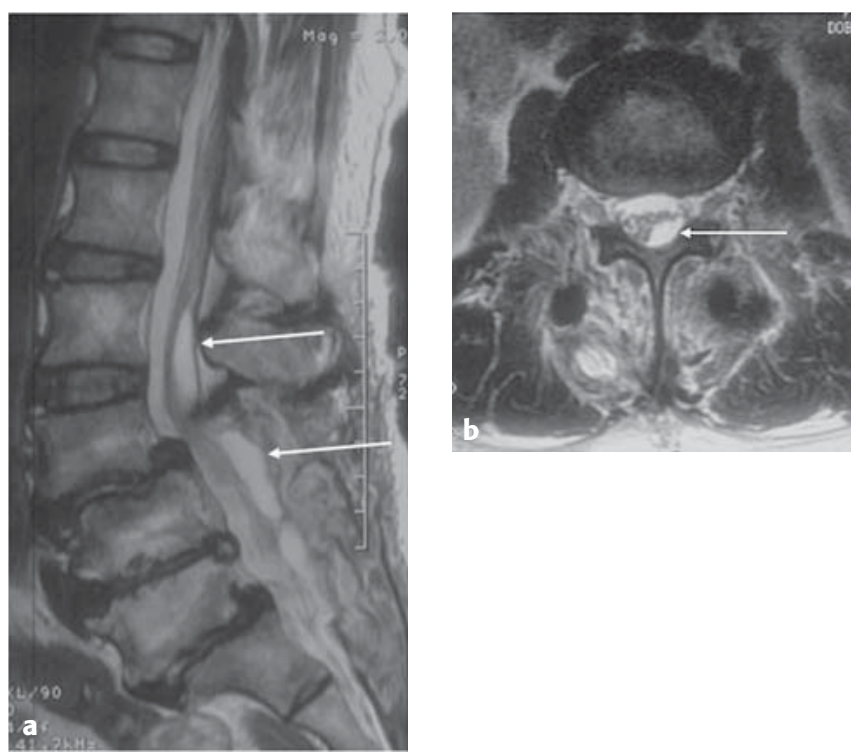

Fig 5 Sagittal T2-weighted magnetic resonance imaging showing disappearance of the subdural hematoma at L2.

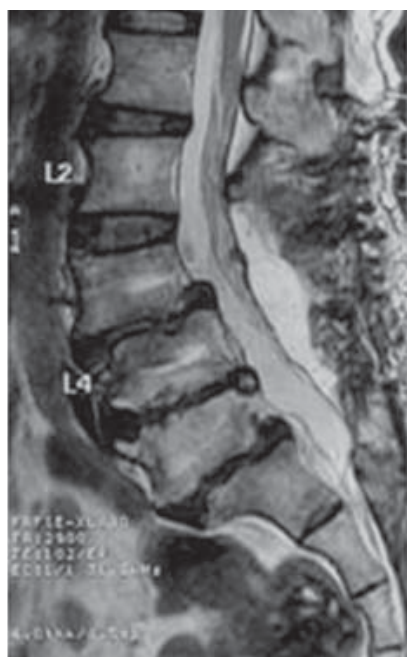




\section{DISCUSSION}

Spinal subdural hematoma is a rare complication of spine surgery with few cases of subacute presentation reported in the literature [1,2]. Both Gehri et al [1] and Reinsel et al [2] reported single-case reports of SSH development, which presented 6 days and 6 weeks following lumbar discectomy, respectively. Their accounts noted clinical presentation of incapacitating leg pain with various degree of neurological deficit $[1,2]$.

Our report is the first, to our knowledge, to note an acute SSH complicating an open lumbar decompressive surgery. Previous reports have proposed various etiological factors associated with SSH [1-7]. Surgery-related trauma has been a factor also noted in cases where the lesion was noted postoperatively [1, 2]. Gehri et al [1] attributed their occurrence of SSH due to a minor dural tear occurred during surgery, which was repaired. Conversely, in their patient, Reinsel et al [2] hypothesized that inadvertent trauma during preoperative myelogram or discectomy had caused SSH. In our case, the patient had no predisposing factors. The dura was adherent to the posterior spinal element and needed blunt dissection during laminectomy, which was traumatic to the delicate dura. However, dissection under magnification might have been less traumatic and avoided the complication.

Immediate diagnosis of SSH is essential. However, in our case, diagnosis was delayed for several reasons. First, a functioning deep drain had made postoperative epidural hematoma compression unlikely. Second, we were confident that there was no residual stenosis after wide laminectomy and decompression of individual nerve roots. Moreover, pedicle screws were inserted under direct visualization of the nerve roots and pedicles, and acceptable postoperative x-rays also made iatrogenic injury less likely. As such, we were prompted to believe that intraoperative manipulation of the neural tissue had caused a neurapraxia and adopted an expectant approach for recovery. However, the neurological deterioration at day 6 prompted us to contemplate of an unusual cause of extensive neurological deterioration following surgery.

To facilitate the diagnosis of SSH, an MRI scan is the preferred imaging modality. In our case, the SSH presented with different signal intensity on MRI, which may have varied because of the age of the clot. This is caused by the changing characteristic when the blood clot progressively liquefied [8]. In our patient, the hematoma was isodense to cerebrospinal fluid at 6 days after the initial surgery. Gel foam, which is used commonly to cover laminectomy defect, can cause considerable confusion on MRI inspection. Blood-soaked gel foam mimics the appearance of an hematoma, but is characteristically not causing thecal sac compression. Furthermore, an axial MRI is useful in differentiating SSH from a postoperative epidural hematoma. An SSH extends within the border of the dura mater and appears smooth. Conversely, an epidural hematoma appears irregular because it is not confined by the dura and extends into the bony recess of the spinal canal [9].

There was considerable delay in surgical intervention in our patient despite the presence of neurological deficit postoperatively. Urgent MRI scan should have been performed, despite the belief of noncompressive neurapraxia to rule out unusual causes. As such, the delay might have jeopardized neurological recovery. Emergency surgical decompression with evacuation of the SSH by durotomy is the treatment of choice for patients presenting with significant neurological deficit, especially with sphincter incontinence [1, 2]. Intraoperatively, the dura might appear deceivingly normal [1], but durotomy confirmed the presence of a subdural hematoma [1].

\section{CONCLUSIONS}

To our knowledge, this case report is the first to note the manifestation of an acute SSH of the lumbar spine following lumbar decompressive surgery. Our case report is meant to raise awareness of the potential occurrence of an acute SSH following lumbar surgery. In addition, spine surgeons should be cognizant that postoperative incapacitating leg pain, unresponsive to routine dose of analgesics and disproportional to surgical trauma outcome, with neurological deterioration is a feature of acute SSH. An MRI is the preferred imaging modality to facilitate in the diagnosis of SSH. Surgery should be performed to decompress the nerve tissues and evacuate SSH for neurological recovery. 


\section{REFERENCES}

1. Gehri R, Zanetti M, Boos N (2000) Subacute subdural haematoma complicating lumbar microdiscectomy. J Bone Joint Surg Br; 82(7):1042-1045.

2. Reinsel TE, Goldberg E, Granato DB, et al (1993) Spinal subdural hematoma: a rare cause of recurrent postoperative radiculopathy. J Spinal Disord; 6(1):62-67.

3. Jain V, Singh J, Sharma R (2008) Spontaneous concomitant cranial and spinal subdural haematomas with spontaneous resolution. Singapore Med J; 49(2):e53-58.

4. Yang MS, Tung YW, Yang TH, et al (2009) Spontaneous spinal and intracranial subdural hematoma. J Formos Med Assoc; 108(3):258261.

5. Chau SY, Tiu SC (2008) Spinal subdural haematoma: a rare complication of low-molecular-weight heparin therapy. Hong Kong Med J; 14(1):64-66.

6. Jonsson LO, Einarsson P, Olsson GL (1983) Subdural haematoma and spinal anaesthesia; a case report and an incidence study. Anaesthesia; 38(2):144-146.

7. Edelson RN, Chernik NL, Posner JB (1974) Spinal subdural hematomas complicating lumbar puncture. Arch Neurol; 31(2):134-137.

8. Kulkarni AV, Willinsky RA, Gray $T$, et al (1998) Serial magnetic resonance imaging findings for a spontaneously resolving spinal subdural hematoma: case report. Neurosurgery; 42(2):398-400; discussion 400-391.

9. Post MJ, Becerra JL, Madsen PW, et al (1994) Acute spinal subdural hematoma: MR and CT findings with pathologic correlates. AJNR Am J Neuroradiol; 15(10):1895-1905.

\section{EDITORIAL PERSPECTIVE}

Chang et al deserve praise for their candor and willingness to share an important-albeit rare-clinical complication with our EBSJ readership. We can all learn from their experience and include subdural postoperative hematoma into our lexicon of differential diagnoses following elective spinal surgery. We suggest that you carefully study the images provided to educate those around us about this occurrence and welcome further discussion and any additional insights by our global multidisciplinary EBSJ readership. The question of an occult dural injury or an indirect intradural vascular injury during manipulation obviously remains [1]. The bigger issue raised by the authors revolves around the controversy of timing of MRI after spine surgery in case of unexpected postoperative neurologic manifestations, such as increased pain, weakness etc. Some degree of neurologic symptoms after major spine surgery is not uncommon. Frequently we tend to abstain from postoperative MRI scans out of fear of confusing artifacts leading to unnecessary intervention. The nature of postoperative MRI artifacts and the timing of their presentation have been poorly studied and warrants further formal investigations to hopefully allay the fear of obtaining this type of test in the future. The threshold when to obtain what type of neuroimaging in case of postoperative examinatino changes - CT, MRI, CT-myelography-is inconsistently applied in the published literature and appears to be subject to personal preferences [2]. We see room for improvement toward greater consistency in our postoperative imaging approaches and a better understanding of relevant versus inconsequential postoperative MRI changes.

1. Goodkin R, Laska LL (1995) Unintended "incidental" durotomy during surgery of the lumbar spine: medicolegal implications. Surg Neurol; 43:4-14.

2. Kostuik JP (2004) Medicolegal consequences of cauda equina syndrome: an overview. Neurosurg Focus; 16(6):39-41. 


\section{COMMENTARY}

Noojan Kazemi, MD

Harborview Medical Center

Seattle, WA, USA

- The case report by Chang and colleagues provides a nice example of a rare postoperative cause of neurological deterioration following lumbar surgery, and in particular following multilevel decompression and fusion.

- Multiple case reviews have reported various etiologies that can result in spinal subdural hematoma (SSH)_including anticoagulation, trauma, post lumbar puncture, and epidural anesthesia. However acute SSH complicating lumbar decompressive surgery is very rare. This article supplements the other two papers describing SSH following lumbar discectomy $[1,2]$.

- The authors noted the previously identified association of a 'minor dural tear' with SSH, but do not specifically declare an unintended durotomy in their own case. They, however, admit to traumatic manipulation of the dura during blunt dissection during laminectomy. This is relevant, as durotomy and CSF leak has been associated with SSH and has been postulated due to alteration of pressures in the dural compartments.

- Although symptoms and signs of a developing SSH can occur up to 6 weeks postoperatively, early recognition and surgical evacuation of the lesion is important for optimal neurological recovery [2].

- The authors acknowledge to considerable delay in both the diagnosis and definitive evacuation of SSH once motor weakness was established on postoperative day 2. This is important as the level of preoperative neurological deficit seemed to be critical for recovery despite prompt surgical evacuation [3]. Consideration should have been given for early intervention once motor weakness had been detected by day 2 .

- Interestingly, severe intractable pain appears to be a clinical feature of acute SSH.

- This case report is useful in raising the awareness of SSH as a potential complication following lumbar decompression surgery and highlights the importance of early diagnosis and intervention.

1. Gehri R, Zanetti M, Boos N (2000) Subacute subdural haematoma complicating lumbar microdiscectomy. J Bone Joint Surg Br; 82(7):1042-1045.

2. Reinsel TE, Goldberg E, Granato DB, et al (1993) Spinal subdural haematoma: a rare cause of recurrent postoperative radiculopathy. J Spinal Disord; 6(1):62-67.

3. Morandi X, Riffaud L, Chabert E, et al (2001) Acute nontraumatic spinal subdural hematomas in three patients. Spine (Phila Pa 1976); 26(23):E547-551. 